\title{
Volume of cerebellar vermis in monozygotic twins discordant for combat exposure: Lack of relationship to post-traumatic stress disorder
}

\section{Citation}

Levitt, James J., Q. Cece Chen, Flavia S. May, Mark W. Gilbertson, Martha E. Shenton, and Roger K. Pitman. 2006. "Volume of Cerebellar Vermis in Monozygotic Twins Discordant for Combat Exposure: Lack of Relationship to Post-Traumatic Stress Disorder." Psychiatry Research: Neuroimaging 148 (2-3) (December): 143-149. doi:10.1016/j.pscychresns.2006.01.013.

\section{Published Version}

doi:10.1016/j.pscychresns.2006.01.013

\section{Permanent link}

http://nrs.harvard.edu/urn-3:HUL.InstRepos:28552593

\section{Terms of Use}

This article was downloaded from Harvard University's DASH repository, and is made available under the terms and conditions applicable to Other Posted Material, as set forth at http:// nrs.harvard.edu/urn-3:HUL.InstRepos:dash.current.terms-of-use\#LAA

\section{Share Your Story}

The Harvard community has made this article openly available.

Please share how this access benefits you. Submit a story.

Accessibility 


\title{
Volume of cerebellar vermis in monozygotic twins discordant for combat exposure: Lack of relationship to post-traumatic stress disorder
}

\author{
James J. Levitta,b,c, Q. Cece Chend, Flavia S. May ${ }^{b, e}$, Mark W. Gilbertson ${ }^{c, f}$, Martha E. \\ Shenton ${ }^{a, b, c}$, and Roger K. Pitman ${ }^{c, e},{ }^{*}$ \\ aVA Boston Healthcare System, Clinical Neuroscience Division, Laboratory of Neuroscience, \\ Brockton, MA 02301, United States \\ bPsychiatry and Behavioral Science Imaging, Surgical Planning Laboratory, MRI Division, \\ Department of Radiology, Brigham and Women's Hospital, Harvard Medical School, Boston, MA, \\ United States \\ 'Department of Psychiatry, Harvard Medical School, Boston, MA, United States \\ dMassachusetts Institute of Technology, Boston, MA, United States \\ eDepartment of Psychiatry, Massachusetts General Hospital, Boston, MA, United States \\ ${ }^{f}$ Research Service, VA Medical Center, Manchester, NH, United States
}

\section{Abstract}

\begin{abstract}
Several functional neuroimaging studies have implicated the cerebellar vermis in post-traumatic stress disorder (PTSD), but there have been no structural neuroimaging studies of this brain structure in PTSD. We utilized magnetic resonance imaging (MRI) with manual tracing to quantify the volumes of three divisions of the mid-sagittal vermis, and their total, within an identical, cotwin control design that employed Vietnam veterans discordant for combat exposure in Vietnam. Each structure's volume was significantly correlated between twins, indicating a partial familial determination: for anterior superior vermis, $r=0.73$; for posterior superior vermis, $r=0.47$; for inferior posterior vermis, $r=0.51$; and for total vermis, $r=0.57$. There were no significant differences between the PTSD and non-PTSD veterans for any vermis volume, and no significant main effects or interactions when their non-combat-exposed co-twins were added to the analyses. Thus, the results do not support the structural abnormality of cerebellar vermis in combat-related PTSD.
\end{abstract}

\section{Keywords}

Cerebellum; Vermis; Stress disorders, post-traumatic; Magnetic resonance imaging; Twins; Monozygotic

\section{Introduction}

The cerebellar vermis, sometimes called the "limbic cerebellum," plays a role in the control of affective behaviors and in the coordination of fear-related somatic and autonomic

\footnotetext{
(C) 2006 Elsevier Ireland Ltd. All rights reserved.

* Corresponding author. MGH-East, Room 2616, Building 149, 13th Street, Charlestown, MA 02129, USA. Tel.: +1 617 726 5333; fax: +1 617 726 4078. roger_pitman@hms.harvard.edu (R.K. Pitman).
} 
conditioned responses (Ghelarducci and Sebastiani, 1997). A review of functional neuroimaging studies of emotion conducted by Reiman (1997) suggests that the vermis participates in the elaboration of normal and pathological forms of anxiety.

Several studies have implicated altered function of the vermis in the pathophysiology of posttraumatic stress disorder (PTSD). Using positron emission tomography in combat veterans with PTSD from recent wars, Pissiota et al. (2002) found greater regional cerebral blood flow $(\mathrm{rCBF})$ during the presentation of combat vs. neutral sounds in the vermis, as well as in the amygdala and periaqueductal gray matter. Anderson et al. (2002) utilized steady-state functional magnetic resonance imaging (fMRI) to assess resting blood flow in the vermis of subjects with repeated childhood sexual abuse and age-matched controls. Abused subjects had higher $\mathrm{T} 2$ relaxation times, indicative of diminished $\mathrm{rCBF}$, than did controls in the vermis, but not in the cerebral or cerebellar hemispheres. According to the authors, these findings suggest that early trauma may interfere with the development of the vermis, and, in turn, inadequate vermis function may fail to contain temporal lobe epilepsy-like psychiatric symptoms found in abused persons. Using single proton emission computed tomography (SPECT), Bonne et al. (2003) found a positive association between regional cerebral blood flow in midline cerebellum and PTSD symptoms.

Teicher et al. (2003) have suggested that the cerebellar vermis should be particularly vulnerable to stress because it has the highest density of glucocorticoid receptors during development, exceeding even those in the hippocampus. There has, however, been very little structural neuroimaging investigation of the vermis in PTSD. In one morphometric study of various brain regions of interest, which did not focus on the vermis, Fennema-Notestine et al. (2002) did not find volumetric differences between victims of intimate partner violence with PTSD and victims without PTSD or normal control subjects in cerebellar gray or white matter regions of interest on structural MRI.

In a recent review, Hull (2002) noted that hippocampal volumetric reduction is the most replicated morphometric finding in PTSD. In a group of monozygotic twins discordant for combat exposure in Vietnam, we also found that combat veterans with PSTD had smaller hippocampi compared to combat veterans without PSTD. This diminished hippocampal volume was shared by the non-combat-exposed, identical co-twins of the combat veterans with PSTD, suggesting that it represents a vulnerability factor for PTSD rather than an acquired PTSD sign (Gilbertson et al., 2002). In the present study, we measured the volume of the cerebellar vermis, divided into three parts, in the same twin subjects as in that study, in order to test the hypothesis that this structure is also reduced in PTSD. Furthermore, should this have turned out to be the case, we planned to use the vermis volumes in the non-combat-exposed co-twins to clarify the origin of the hypothesized vermis volume reduction.

\section{Materials and methods}

\subsection{Subjects}

Subjects were members of 48 male monozygotic (identical) twin pairs discordant for combat exposure in Vietnam. This means that within each pair, one "exposed" twin had participated in military combat, whereas his unexposed co-twin had not. Among the exposed twins, 24 met DSM-IV criteria for current combat-related PTSD, and 24 had never met criteria for combatrelated PTSD (non-PTSD). The means of ascertainment and recruitment of the subjects, as well as information regarding their combat severity, frequency of non-combat traumatic events, presence of other mental disorders, and substance use, have been presented in detail elsewhere (Gilbertson et al., 2002; Orr et al., 2003). 
As previously noted (Gilbertson et al., 2002), subects were excluded if they met DSM-IV criteria for a psychotic/affective disorder or non-combat-related PTSD. Due to the high comorbidity of major depression and substance use disorders with PTSD, these disorders did not represent exclusion criteria. History of major neurologic disorder or significant abnormality discovered upon MRI scan during the course of the study which could impact volumetric measurements, e.g., tumor, resulted in exclusion of two subjects; the affected individuals were provided with this information and arrangements made for appropriate clinical transfer of MRI reports. Three subjects were unable to complete MRI due to claustrophobic reactions, and one subject due to a cardiac condition. Two MRI scans were not segmented for volumetric measurement due to excessive movement artifact.

The same MRI scans that were used to quantify hippocampal volume in the study of Gilbertson et al. (2002) were used to quantify cerebellar vermis volume here. That is, the subjects in the present study are the same as those in that report, with a few exceptions. Whereas the Gilbertson et al. study's data analysis approach (analysis of variance) discarded both members of a twin pair if data were missing from one of its members, the mixed model statistical technique used in the present study allowed for the inclusion of data from singletons (i.e., subjects whose twin's data were missing). Additionally, a few subjects were lost for the present study due to technically inadequate scans (see below).

The psychodiagnostic and neuroimaging procedures were described in detail in a written informed consent document that was approved by the relevant Institutional Review Boards. This document was provided to the subject to read, and further explained by a doctoral-level psychologist, who then answered any questions. All subjects signed the consent document prior to participation.

\subsection{PTSD diagnosis}

The Clinician-Administered Post-Traumatic Stress Disorder Scale (CAPS; Weathers et al., 2001) was used to make a categorical diagnostic determination of the presence or absence of combat-related PTSD (current vs. never had) in the exposed twin. In our hands, the interrater, test-retest reliability of total CAPS score is 0.94 (intra-class correlation coefficient). Due to ambiguous predictions, combat-exposed subjects with past but not current PTSD, and their cotwins, were excluded.

\subsection{MRI acquisition and processing}

The MRI acquisition protocol and the post-processing of images have been previously described (Gilbertson et al., 2002). Briefly, we obtained 1.5-mm-thick coronal slices that were derived from a series of contiguous slices using an SPGR sequence (i.e., repetition time $=35 \mathrm{~ms}$, echo time $=5 \mathrm{~ms}$, voxel dimensions $=0.9375 \times 0.9375 \times 1.5 \mathrm{~mm}$ ). An anisotropic diffusion filter ( $k=13$ for SPGR and 90 for proton/T2 images, iteration=3) was applied to the images to reduce noise prior to processing each set of scans.

All cerebellar images were initially aligned in order to compensate for head tilt and rotation during the MRI acquisition. In order to align all scans uniformly along the same midline axes, we chose intrinsic cerebellar landmarks, rather than using cortical landmarks, because cerebellar and cortical alignment is not uniform (Courchesne et al., 1994). Lines were drawn at the midline on axial and coronal plane slices at two separate levels and on the sagittal plane at one level. On the axial plane, superiorly, we chose the slice that showed the midbrain-pontine junction in which the division between right and left cerebral crus, the interpendular fossa, completely disappeared. At this level, we drew a line connecting the apex of anterior and posterior convexities of the vermis. More inferiorly, we chose the slice in which both lateral recesses of the fourth ventricle divided the rostral medulla from the cerebellar hemispheres. 
At this level, we drew a second line connecting the anterior and posterior peak points of the vermis. On the coronal plane, posteriorly, we chose the slice in which the anterior lobe of the vermis first appeared within the primary fissure, viewed sagittally. We then drew a first line to pass through the apex of the anterior lobe of the vermis, the apex of the convexity of the primary fissure (seen bilaterally), and the apex of the inferior convexity of the cerebellar hemispheres. Proceeding anteriorly, we chose the next slice to be the first slice in which the fourth ventricle was shown to connect over the superior aspect of the nodulus. A second line then was drawn to connect the superior apex of the vermis, the superior apex of the nodulus, and the division between the two tonsils.

Following the above, a computer program script was run that realigned and resampled our slices into isotropic voxels based upon the information derived from the lines at the midlines traced in the axial and coronal cerebellar planes. The final step was to draw a line at the midline in the sagittal plane. Here we used the conventional landmarks of the anterior and posterior commissures. We selected the sagittal slice in which the cerebral aqueduct could be seen most clearly. Then we drew a single line connecting the center of the anterior commissure with a point slightly beyond the center of the posterior commissure. Lastly, a second computer program script was run that realigned and resampled the slices, again into isotropic voxels, now based on midline information from all three planes. The voxel size was set to the smallest dimension of the original voxels, which was $0.9375 \times 0.9375 \times 0.9375 \mathrm{~mm}$ (Hirayasu et al., 2000).

\subsection{Volumetric calculations of vermis and its divisions}

The vermis was parcellated into three combined gray and white matter lobule regions, viz., anterior superior, posterior superior, and inferior posterior lobules (or lobules 1-V, VI-VII and VIII-X respectively), based upon the methods described in our prior study in schizophrenia (Levitt et al., 1999). Viewing the vermis in the sagittal plane, we manually traced the primary and prepyramidal fissures from the surface of the vermis to the apex of the fourth ventricle. In the present study, we performed these tracings on three sagittal slices. We chose the mid-sagittal slice, at the level of the vermis, and then included parasagittal slices on either side (see Fig. 1). Our selection criteria for the mid-sagittal, and parasagittal slices were based upon the best fit of several criteria: 1 ) the mid-sagittal slice was that slice where the cerebral aqueduct was most clearly visualized; 2) the three middle slices were those slices where the vermian corpus medullare, or white matter, retained its characteristic shape (Press et al., 1989); and 3) the three middle slices were those where the outer contour of the vermis was most characteristically vermal (Courchesne et al., 1989).

Our methods differed in two ways from our prior study (Levitt et al., 1999). First, in our prior study we segmented the cerebellar hemispheres and vermis into gray and white matter using an iterative expectation-maximization segmentation program, which was based upon seed points manually selected for different cerebellar tissues (Wells et al., 1996). In the present study, we did not quantify vermis gray and white matter separately. Second, in the prior study, we essentially traced the full lateral extent of the vermis. In the present study, because we had first oriented the vermis slices into a uniform alignment, we did not believe it was necessary to perform this challenging and time-consuming task. Instead, we uniformly chose three midsagittal vermis slices for all cases, whereas in our prior study there was no set number of slices per case, and, generally, more than three times as many sagittal slices per case were measured. A second rater performed the same volumetric analyses on 27 of the subjects (randomly selected) in order to establish interrater reliability. 


\subsection{Statistical analysis}

Data were analyzed by means of a mixed model (Little et al., 1996) that treated PTSD Diagnosis in the combat-exposed twin (present or absent), as a between-pairs fixed effect, Combat Exposure as a within-pairs fixed effect, and pairs as a random effect. The mixed model analysis yields a $t$ statistic with one degree of freedom (not to be confused with Student's $t$ ). If a dependent variable represents a vulnerability factor for PTSD, the model predicts a significant Diagnosis effect in the absence of a Diagnosis by Exposure interaction. If, on the other hand, a dependent variable represents an acquired PTSD sign, the model predicts a significant Diagnosis by Exposure interaction. Student's $t$-tests were performed on the volumetric data from the PTSD vs. non-PTSD, combat-exposed twins. Pearson product-moment correlations were also performed as described below.

\section{Results}

\subsection{Missing data}

MRI data were unavailable in 4 combat-exposed, PTSD subjects for the following reasons: two had shrapnel that prohibited scanning, one developed an acute medical condition that precluded scanning, and one developed claustrophobia in the scanner. MRI data were generally unusable in one exposed twin without PTSD who had a brain tumor, and in one unexposed cotwin of an exposed twin with PTSD, who had had past brain surgery. MRI data were specifically unusable for technical reasons for the purpose of quantifying vermis volume in two additional exposed twins with PTSD, one additional exposed twin without PTSD, three additional unexposed co-twins of exposed twins with PTSD, and one additional unexposed co-twin of an exposed twin without PTSD.

\subsection{Demographics, psychometrics, and whole brain volumes}

Table 1 provides subjects' demographic and psychometric measures, and whole brain volume, along with the results of statistical analyses. As would be expected, combat-related CAPS scores were higher in the combat-exposed subjects with PTSD than in the combat-exposed subjects without PTSD. Otherwise, there were no significant main effects or interactions, except for an uninterpretable Diagnosis $\times$ Exposure interaction for whole brain volume, which was not accompanied by a significant difference between the combat-exposed twins with vs. without PTSD $(t(38)=-0.6, P=0.54)$.

\subsection{Vermis volumes}

3.3.1. Interrater reliabilities-Interrater reliabilities for vermis volumes were as follows: anterior superior 0.86 , posterior superior 0.77 , inferior posterior 0.90 , total vermis 0.90 (intraclass correlation coefficients).

3.3.2. Results in combat-exposed twins-Group means for the PTSD and non-PTSD, combat-exposed twins are presented in Table 1 . There were no significant differences in vermis volumes between the combat-exposed twins with vs. without PTSD: anterior superior $(t(38)$ $=-0.2, P=0.84$, difference between means $=-0.01 \mathrm{ml}, 95 \% \mathrm{CI}:-0.11 \mathrm{ml}, 0.09 \mathrm{ml})$; posterior superior $(t(38)=-0.4, P=0.73$, difference between means $=-0.02 \mathrm{ml}, 95 \%$ CI: $-0.14 \mathrm{ml}, 0.10 \mathrm{ml})$; inferior posterior $(t(38)=-0.3, P=0.80$, difference between means $=-0.01 \mathrm{ml}, 95 \%$ CI: $-0.09 \mathrm{ml}$, $0.07 \mathrm{ml})$; total vermis $(t(38)=-0.6, P=0.58$, difference between means $=-0.06 \mathrm{ml}, 95 \%$ CI: $-0.28 \mathrm{ml}, 0.16 \mathrm{ml}$ ). Estimated effect sizes (Cohen's $d$ ) were: anterior superior $-0.07 d$; posterior superior $-0.11 d$; inferior posterior $-0.08 d$; total vermis $-0.18 d$. Prospective power analyses based upon these estimated effect sizes indicate that the sample sizes in each of two combatexposed groups (PTSD, non-PTSD) that would be required to detect a significant group difference for total vermis would be 486 , with even larger samples required for each division. 
3.3.3. Results adding non-combat-exposed twins-Group means for the non-combatexposed co-twins are also presented in Table 1, as are results of the mixed-model analyses incorporating both combat-exposed and non-combat exposed twins. As will be seen, there were no main significant main effects or interactions. Table 1 also presents estimated differences in means derived from the mixed model analyses and their $95 \%$ confidence intervals.

3.3.4. Volumetric correlations between twins-Pearson product-moment correlations between vermis volumes in combat-exposed and non-combat-exposed twins collapsed across diagnostic groups were: anterior superior $r=0.73(P<0.0001)$, posterior superior $r=0.47$ $(P=0.003)$, inferior posterior $r=0.51(P=0.001)$, total vermis $r=0.57(P<0.001)$.

3.3.5. Volumetric-psychometric correlations-Pearson product-moment correlations between combat veterans' vermis volumes and their CAPS scores collapsed across diagnostic groups were: anterior superior $r=-0.10, P=0.54$; posterior superior $r=-0.02, P=0.90$; inferior posterior $r=-0.04, P=0.80$; total vermis $r=-0.07, P=0.67$. These correlations remained nonsignificant when analyzed separately in the PTSD and non-PTSD, combat-exposed groups.

\section{Discussion}

The highly significant correlation between twins for the volume of total mid-sagittal vermis and each division separately indicate that the size of these brain structures is under partial familial influence. However, in the absence of dizygotic twin subjects, it is not possible to conclude whether this reflects heredity or shared environment.

The results do not support reduced volume of the midsagittal cerebellar vermis, or any of its three divisions that were measured, in combat-related PTSD. With regard to the possibility of Type II error, the results allow us to conclude with $95 \%$ confidence that the total vermis volume of PTSD combat veterans could be no more than $9 \%$ smaller than that of non-PTSD combat veterans ( $8 \%$ for anterior superior, $16 \%$ for posterior superior, and $9 \%$ for inferior posterior). The prospective power analyses indicate that a prohibitive number of subjects would be required to confirm these small trends. Even if they were to be confirmed, they would likely be so small as to be of dubious clinical significance. A possible but unlikely source for our failure to show significant group differences may be that we did not parcellate the vermis into gray and white matter. This, however, is difficult to do because the small size of cerebellar folia, compared to the gyri in the cortical mantle, creates a resolution challenge, with partial voluming, either for manual tracing or for more automatic segmentation programs.

In the absence of any significant differences between combat veterans with and without PTSD, it is not surprising that the results in their identical co-twins were uninformative.

The absence of observed, gross anatomic structural differences does not preclude the possibility of functional abnormalities in the cerebellar vermis in PTSD, which may deserve further study despite these negative morphometric findings. The present results also do not rule out the possibility that early life trauma could affect vermal volume.

\section{Acknowledgments}

This work was supported by a Department of Veterans Affairs Merit Review Grant to Dr. Gilbertson, USPHS Grant \#K02-MH01110 to Dr. Shenton, and USPHS Grant \#R01-MH54636 to Dr. Pitman. 


\section{References}

Anderson CM, Teicher MH, Polcari A, Renshaw PF. Abnormal T2 relaxation time in the cerebellar vermis of adults sexually abused in childhood: potential role of the vermis in stress-enhanced risk for drug abuse. Psychoneuroendocrinology 2002;27:231-244. [PubMed: 11750781]

Bonne O, Gilboa A, Louzoun Y, Brandes D, Yona I, Lester H, Barkai G, Freedman N, Chisin R, Shalev AY. Resting regional cerebral perfusion in recent posttraumatic stress disorder. Biological Psychiatry 2003;54:1077-1086. [PubMed: 14625150]

Courchesne E, Press GA, Murakami J, Berthoty D, Grafe M, Wiley CA, Hesselink JR. The cerebellum in sagittal plane-anatomic-MR correlation: 1. The vermis. American Journal of Roentgenology 1989;153:829-835. [PubMed: 2773740]

Courchesne E, Yeung-Courchesne R, Egaas B. Methodology in neuroanatomic measurement. Neurology 1994;44:203-208. [PubMed: 8309559]

Fennema-Notestine C, Stein MB, Kennedy CM, Archibald SL, Jernigan TL. Brain morphometry in female victims of intimate partner violence with and without posttraumatic stress disorder. Biological Psychiatry 2002;52:1089-1101. [PubMed: 12460692]

Ghelarducci B, Sebastiani L. Classical heart rate conditioning and affective behavior: the role of the cerebellar vermis. Archives Italiennes de Biologie 1997;135:369-384. [PubMed: 9270898]

Gilbertson MW, Shenton ME, Ciszewski A, Kasai K, Lasko NB, Orr SP, Pitman RK. Smaller hippocampal volume predicts pathologic vulnerability to psychological trauma. Nature Neuroscience 2002;5:1242-1247.

Hirayasu Y, McCarley RW, Salisbury DF, Tanaka S, Kwon JS, Frumin M, Snyderman D, YurgelunTodd D, Kikinis R, Jolesz FA, Shenton ME. Planum temporale and Heschl gyrus volume reduction in schizophrenia: a magnetic resonance imaging study of first-episode patients. Archives of General Psychiatry 2000;57:692-699. [PubMed: 10891040]

Hull AM. Neuroimaging findings in post-traumatic stress disorder: systematic review. British Journal of Psychiatry 2002;181:102-110. [PubMed: 12151279]

Levitt JJ, McCarley RW, Nestor PG, Petrescu C, Donnino R, Hirayasu Y, Kikinis R, Jolesz FA, Shenton ME. Quantitative volumetric MRI study of the cerebellum and vermis in schizophrenia: clinical and cognitive correlates. American Journal of Psychiatry 1999;156:1105-1107. [PubMed: 10401463]

Little, RC.; Miliken, GA.; Stroup, WW.; Wolfinger, RD. SAS System for Mixed Models. SAS Institute Inc.; Cary, NC: 1996.

Orr SP, Metzger LJ, Lasko NB, Macklin ML, Hu FB, Shalev AY, Pitman RK. Physiologic responses to sudden, loud tones in monozygotic twins discordant for combat exposure: association with PTSD. Archives of General Psychiatry 2003;60:283-288. [PubMed: 12622661]

Pissiota A, Frans O, Fernandez M, von Knorring L, Fischer H, Fredrikson M. Neurofunctional correlates of posttraumatic stress disorder: a PET symptom provocation study. European Archives of Psychiatry and Clinical Neuroscience 2002;2:68-75. [PubMed: 12111339]

Press GA, Murakami J, Courchesne E, Berthoty DP, Grafe M, Wiley CA, Hesselink JR. The cerebellum in sagittal plane-anatomic-MR correlation: 2. The cerebellar hemispheres. American Journal of Roentgenology 1989;153:837-846. [PubMed: 2773741]

Reiman EM. The application of positron emission tomography to the study of normal and pathologic emotions. Journal of Clinical Psychiatry 1997;58S16:4-12. [PubMed: 9430503]

Teicher MH, Andersen SL, Polcari A, Anderson CM, Navalta CP, Kim DM. The neurobiological consequences of early stress and childhood maltreatment. Neuroscience and Biobehavioral Reviews 2003;27:33-44. [PubMed: 12732221]

Weathers FW, Keane TM, Davidson JR. Clinician-administered PTSD scale: a review of the first ten years of research. Depression and Anxiety 2001;13:132-156. [PubMed: 11387733]

Wells WM III, Grimson WEL, Kikinis R, Jolesz FA. Adaptive segmentation of MRI data. IEEE Transactions in Medical Imaging 1996;15:429-442. 

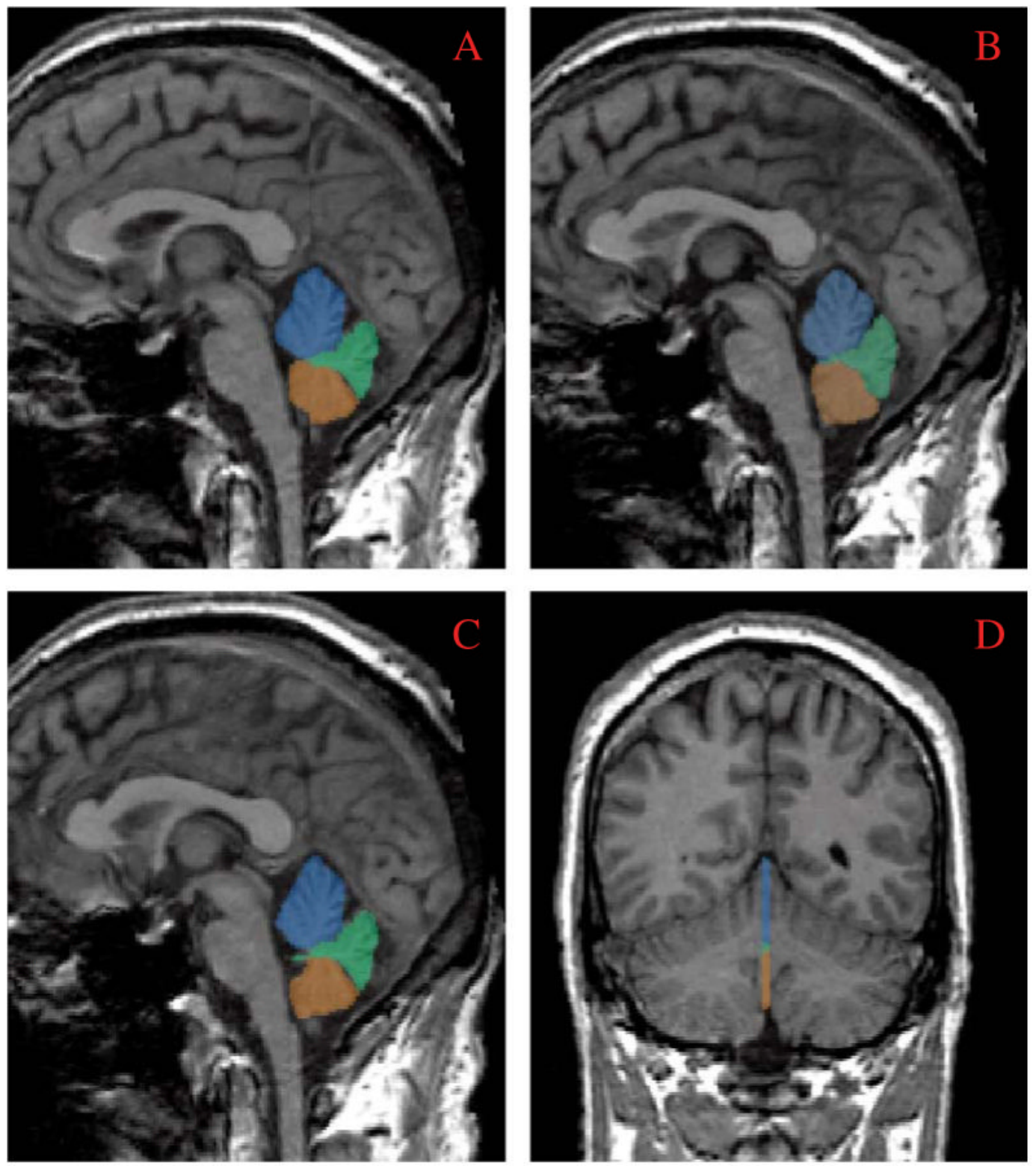

Fig. 1.

Panes A-C demonstrate manual tracings performed on the three sagittal slices of the cerebellar vermis, including a mid-sagittal (B) and 2 parasagittal slices (A and C). Pane D demonstrates all three slices in a coronal view. Parcellated areas included anterior superior vermis (lobules I-V) appearing in blue; posterior superior vermis (lobules VI-VII) in green: and inferior posterior vermis (lobules VIII-X) in orange. See text for a description of the anatomic criteria used for the parcellations. 
Levitt et al.

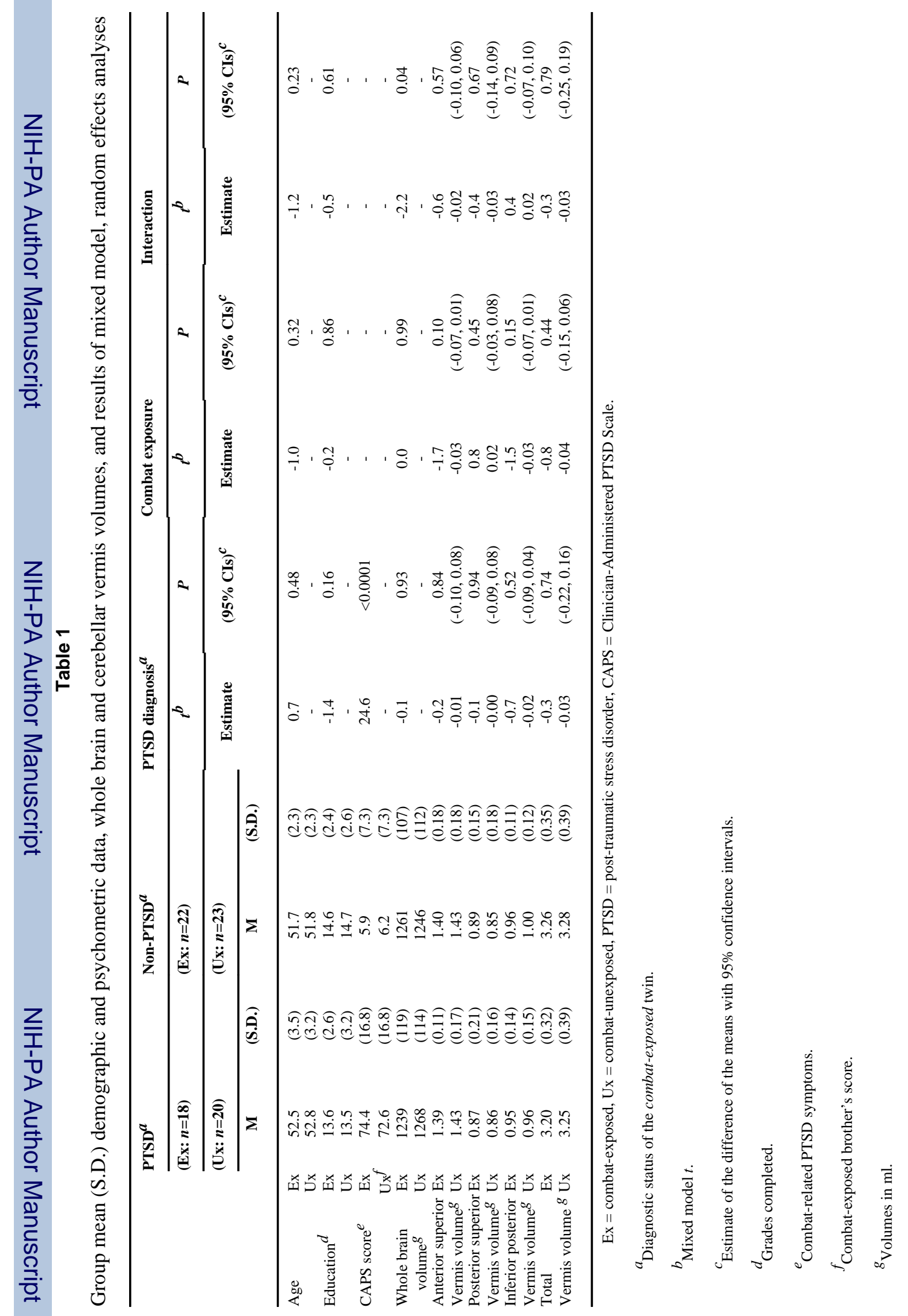

